\title{
Effectiveness of a multiple intervention programme for the prevention of falls in older adults persons from a University of the Third Age
}

Vilmar Mineiro da Silva' $\mathbb{1}$ Alex Sandro Faria de Arruda' (ID Laís dos Santos Vinholi e Silva' ${ }^{1 D}$ Francisco Luciano Pontes Junior? $\mathbb{D}$ Meire Cachioni ${ }^{1,2}$ (I) Ruth Caldeira de Melol (D)

\section{Abstract}

Objective: To evaluate the effectiveness of a multiple intervention programme for the prevention of falls in older adults from a University of the Third Age (U3A). Method: A quasi-experimental, non-controlled, longitudinal and quantitative study was performed. 69 older adults were allocated into three groups: Control (CG), Physical Exercise (PEG) and Multiple Intervention (MIG). The instruments/tests used were: sociodemographic questionnaire, Geriatric Depression Scale (15-items), Mini-Mental State Examination, Timed-Up and Go (TUG), Sit-to-Stand and Hand-Grip Strength, Falls Efficacy ScaleInternational and Falls Risk Awareness Questionnaire (FRAQ).The PEG and MIG groups underwent physical training (walking, muscular resistance, and balance) for 16 weeks (2x/week, $60 \mathrm{~min} / \mathrm{session})$. In the same period, the MIG also participated in educational sessions (1x/week, 60min/session). Covariance analysis was used for group comparisons. The effect size of the interventions was also calculated. The level of significance was set at $p<0.05$. Results: 51 older adults (67 \pm 6.2 years and $76.3 \%$ women), of whom 15 were in the CG, 20 in the PEG and 16 in the MIG, concluded the study. TUG time in both intervention groups was reduced, but FRAQ score improved in the MIG only. Both interventions had a small effect on TUG time, while multiple intervention had a large effect on FRAQ. Conclusion: Multiple intervention brought additional benefits to the older adults from this U3A. In addition to improving balance, the older adults who underwent the multiple intervention increased their knowledge about risk factors for falls.

\footnotetext{
Universidade de São Paulo, Escola de Artes, Ciências e Humanidades, Programa de Pós-graduação em Gerontologia. São Paulo, SP, Brasil.

2 Universidade Estadual de Campinas, Faculdade de Ciências Médicas, Programa de Pós-graduação em Gerontologia. Campinas, SP, Brasil.
}

Research funding: National Council for Scientific and Technological Development (or CNPq), Universal Bid Notice (process no 428865/2016-0) and Coordination for the Improvement of Higher Education Personnel (or CAPES), Financing Code 001 - Brazil.

The authors declare there are no conflicts of interest in relation to the present study.

Correspondence

Ruth Caldeira de Melo

Keywords: Health of the Elderly. Accident Prevention. Accidental Falls. Exercise Movement Techniques. Physical Fitness. Health Education. 


\section{INTRODUCTION}

Falls in the older adults are a major public policy concern, mainly due to the negative outcomes and high health spending associated with their consequences ${ }^{1,2}$. In Brazil, the prevalence of falls among communitydwelling older adults is approximately $25 \%$, and is higher among women and older individuals ${ }^{3}$. Since falls have multifactorial characteristics, i.e., several risk factors may be involved in a single event ${ }^{1}$, it is key that preventive programs include different types of intervention ${ }^{4}$.

Preventive interventions can be administered alone or in combination. The latter can be designed and offered individually based on the assessment of risk factors for each older adults (multifactorial intervention) or generalized and similar for all participants (multiple intervention) ${ }^{4}$. Scientific evidence suggests that multifactorial intervention and physical exercise (a single intervention) are the most effective in reducing falls, while the latter seems to have the greatest effect in reducing risk ${ }^{4,5}$. It is recommended that interventions involving physical training include balance and muscular endurance exercises, either for the older adults in general or for those at risk of falls ${ }^{5}$.

Among the combined interventions, multifactorial interventions also seem to be the most effective in reducing falls, followed by multiple actions that combine physical exercises with educational activities or environmental modifications ${ }^{6}$. Therefore, multifactorial intervention should be the first option in offering this type of service to the older adults ${ }^{6}$. As multifactorial interventions are complex and require the involvement of more professionals ${ }^{4,7}$, offering them more to the wider population is often costly and infeasible.

Although multiple interventions are not as effective as multifactorial interventions in preventing falls ${ }^{6}$, Goodwin et al. ${ }^{8}$ showed that, compared to a control situation, multiple intervention was also able to reduce the number of older adults who fell and the rate of falls, and physical exercise is an important component in achieving such results ${ }^{4}$. Other approaches widely used in multiple interventions include drug optimization, environmental modifications, and educational interventions ${ }^{7}$. In terms of educational interventions, their role in preventing falls is not yet fully established ${ }^{4}$. However, Schepens et al. ${ }^{9}$ suggest that they may contribute to improving the knowledge of the older adults about risk factors and, consequently, to the adoption of preventive behaviors.

Given the above, the present study aimed to evaluate the effectiveness of physical training, combined (multiple intervention) or not (isolated intervention) with educational intervention, on the physical-functional performance, self-efficacy and perception of risk factors for falls in older adults participants of an Open University for the Third Age (or U3A).

\section{METHOD}

A quasi-experimental, non-controlled longitudinal and quantitative study was carried out at the U3A of the School of Arts, Sciences and Humanities of the Universidade de São Paulo (or EACH | USP). Characterized as a continuing education program for refreshing and acquiring new knowledge, this U3A receives about 300 older adults every six months, who can enroll for free in some of the regular subjects of the University's undergraduate courses, didacticcultural activities and physical-sports activities. For the present study, we recruited older adults enrolled in the sport-physical activity entitled "Get Balanced" from February 2017 to December 2018.

The sample size calculation was performed using the G*Power software (version 3.1.9.4) using the intragroup comparison method (repeated measures) based on the values of the Timed Up and Go test (TUG) $[7.3$ ( \pm 1.0$)$ seconds] and Sit-To-Stand test (STS) $[14.6( \pm 2.0)$ seconds], previously reported in the literature for older adults participants of the $\mathrm{U}^{3} \mathrm{~A}^{10}$. Considering a type I error of $5 \%$ and type II error of $20 \%$, i.e. $80 \%$ sample power, 57 individuals (19 per group) would be needed to detect $10 \%$ intragroup differences in both tests. In order to reduce the effects of possible losses, a margin of $20 \%$ was added to the sample calculation, leading to a final amount of 23 people per group. 
Ninety-five older adults men and women enrolled in the U3A volunteered to participate in the study and were selected according to the following inclusion criteria: 60 years of age or older, good health, and medical clearance for performing physical exercises. The study excluded older adults people who presented any health problem that contraindicate the participation in group physical exercises, such as those with diseases and/or limiting chronic conditions (cardiorespiratory diseases, neurological diseases, diagnosed vestibular disorders, musculoskeletal disorders and diagnosed cognitive impairment). The older adults with poor vision, a history of recurrent falls (more than two events in 12 months) and who used walking aids were also excluded from the study. After applying these criteria, 69 older adults were included and allocated by convenience in three experimental groups: Control (CG, $n=23)$, Physical Exercise (PEG, $n=25$ ) and Multiple Intervention (MIG, $\mathrm{n}=21$ ).

The older adults placed in the CG were instructed to maintain their routine activities and not initiate any kind of physical training while participating in the study. At the end of the 16-week follow-up period, the CG older adults were invited to participate in the same physical training program offered to the other groups. The older adults from the PEG underwent a multimodal physical training protocol, consisting of resistance, aerobic endurance/walking and balance exercises, twice a week with 60 minutes each session for 16 weeks. The older adults from the MIG were submitted to the same physical training protocol offered to the PEG (twice a week, 60 minutes/ session for 16 weeks), which was combined with an educational intervention protocol for fall prevention (once a week, 60 minutes/ session for 16 weeks).

The educational intervention was conducted in order to increase the knowledge of the older adults about risk factors and the prevention of falls. Each session was divided into two parts: lecture (30 minutes) and coordinated discussion to share experiences and clarify the doubts of participants (30 minutes). The topics covered in the educational sessions included important information for fall prevention, such as fall definition, risk factors and major preventive interventions. A detailed description of the physical training protocols and educational intervention can be seen in Chart 1 .

Chart 1. Description of the characteristics of the multimodal physical training and educational intervention protocols used in the study. São Paulo, 2018.

\begin{tabular}{|l|l|l|}
\hline \multirow{2}{*}{ Characteristics } & Protocols \\
\cline { 2 - 3 } & Multimodal Physical Training & Educational Intervention \\
\hline $\begin{array}{l}\text { Experimental } \\
\text { Group }\end{array}$ & $\begin{array}{l}\text { Physical Exercise (PEG) and Multiple } \\
\text { Intervention (MIG) }\end{array}$ & Multiple Intervention (MIG) \\
\hline Local & $\begin{array}{l}\text { Sports gymnasium (more specifically, sports } \\
\text { court, bodily activity room and surroundings). }\end{array}$ & $\begin{array}{l}\text { Meeting room equipped with two rectangular } \\
\text { tables and chairs. }\end{array}$ \\
\hline Materials & $\begin{array}{l}\text { Chairs, anklets (1 to } 3 \mathrm{~kg}), \text { steps, poles, balls of } \\
\text { various sizes, mats, cones and balance discs. }\end{array}$ & Microcomputer and video projector. \\
\hline
\end{tabular}

to be continued 
Continuation of Chart 1

\begin{tabular}{|c|c|c|}
\hline \multirow[t]{2}{*}{ Characteristics } & \multicolumn{2}{|l|}{ Protocols } \\
\hline & Multimodal Physical Training & Educational Intervention \\
\hline $\begin{array}{l}\text { Activities and } \\
\text { Exercises }\end{array}$ & $\begin{array}{l}\text { (1) aerobic endurance: indoor (outdoor) or } \\
\text { outdoor (campus) walking; } \\
\text { (2) resistance: lower limb exercises performed } \\
\text { in sitting (chair) and/or standing position } \\
\text { (plantar flexion and ankle dorsiflexion, } \\
\text { knee flexion and extension, hip flexion, hip } \\
\text { abduction and squatting /sitting and rising } \\
\text { from the chair); } \\
\text { (3) balance: exercises involving static and } \\
\text { dynamic postures (single-legged support, } \\
\text { tandem, cone zigzag, toe-walking, heel- } \\
\text { walking, lateral-walking, obstacle-walking and } \\
\text { ludic activities*). }\end{array}$ & $\begin{array}{l}\text { Each session was divided into two parts: } \\
\text { 1) lecture ( } 30 \text { minutes) and 2) coordinated } \\
\text { discussion for sharing experiences and clarifying } \\
\text { questions from participants ( } 30 \text { minutes). The } \\
\text { themes developed during the intervention } \\
\text { included: falls (definition, consequences, risk } \\
\text { behaviors and prevention); diseases and changes } \\
\text { in health (major diseases that predispose the } \\
\text { individual to falls and how to prevent them); } \\
\text { extrinsic factors (such as improving the home } \\
\text { environment, better footwear and foot care); } \\
\text { reducing the risk of falling (most recommended } \\
\text { physical exercise); and other factors influencing } \\
\text { falls (drug interactions, diet, cognition and fear } \\
\text { of falling). }\end{array}$ \\
\hline Frequency & Twice per week. & Once a week. \\
\hline Duration & $\begin{array}{l}60 \mathrm{~min} / \mathrm{session} \text { (i.e. } 20 \mathrm{~min} / \mathrm{session} \text { for each } \\
\text { multimodal training component). }\end{array}$ & $\begin{array}{l}60 \mathrm{~min} / \mathrm{session} \text { (i.e. } 30 \mathrm{~min} / \mathrm{session} \text { for lecture } \\
\text { and } 30 \mathrm{~min} / \text { session for discussion). }\end{array}$ \\
\hline $\begin{array}{l}\text { Volume and } \\
\text { Intensity }\end{array}$ & $\begin{array}{l}\text { (1) moderate-intense walking, according to the } \\
\text { subjective perception of effort; } \\
\text { (2) two to three sets of } 10 \text { to } 15 \text { repetitions, } \\
\text { with a load of } 1 \text { to } 3 \mathrm{~kg} \text { (anklet) and rest } \\
\text { interval of one minute. Volume and load } \\
\text { increments were performed when volunteers } \\
\text { rated the intensity of effort as mild; } \\
\text { (3) increased difficulty individually, from } \\
\text { reductions in upper limb support, reductions in } \\
\text { support base, inclusion of unstable surfaces and } \\
\text { association with cognitive tasks (verbal fluency, } \\
\text { mental calculations and immediate memory). }\end{array}$ & Not applicable. \\
\hline
\end{tabular}

*Every 15 days the balance exercises were replaced by ludic activities involving body displacement and other motor and/or cognitive skills (examples: adapted volleyball, dodgeball, adapted tic-tac-toe and body perception/expression activities).

Data were collected by a team of researchers (graduate students and academics) who were previously trained and familiar with the tests and measuring instruments. The data were collected through the completion of a form containing sociodemographic information (age, gender, marital status and education), anthropometric data (weight, height and body mass index (BMI)) and history of falls in the last 12 months. Weight and height were measured with the aid of a mechanical scale and a wall stadiometer, respectively. The ratio between weight (in kilograms, $\mathrm{kg}$ ) and height squared (in meters, $\mathrm{m})$ was used to calculate BMI $\left(\mathrm{kg} / \mathrm{m}^{2}\right)$. To better characterize the sample, depressive symptoms and cognitive impairment were assessed respectively using the 15-item Geriatric Depression Scale (GDS) and Mini Mental State Exam (MMSE), using versions adapted and validated for the Brazilian population in previous studies ${ }^{11-13}$.

The 15-item GDS is widely used to screen depressive symptoms in the older adults. For the Brazilian population, the $5 / 6$ score (not case/case) showed good sensitivity ( $81 \%$ ) and specificity (71\%) for the diagnosis of depression, as well as presenting satisfactory reliability for clinical use $\mathrm{e}^{11,12}$. 
The MMSE is a cognitive screening instrument that assesses five areas of cognition: orientation; registration; attention and calculation; recall and language. Although the MMSE is widely used in Brazil, there are still differences regarding cutoff scores and some of its psychometric characteristics ${ }^{14}$. The present study used the version suggested by Brucki et al. ${ }^{13}$.

The outcome variables were TUG, handgrip strength (HGS), and STS scores, as well as scores in the Falls Efficacy Scale-International (FES-I) and Falls Risk Awareness Questionnaire (FRAQ).

The TUG test was used to assess mobility and balance. This test measures the time taken to get up from a chair with arms, walk three meters forward, turn around, walk back, and sit down in the chair ${ }^{15}$. For older adults Brazilians, times longer than 12.47 seconds (usual speed) indicate a higher risk of falls ${ }^{16}$. The volunteers were allowed to take the course once prior to timing to familiarize them with the test. The older adults were then instructed to move as quickly as possible, provided that their safety was not endangered. The test was performed three times, with the average time spent in all attempts used in the analyzes, provided that the variability between the measurements was less than $10 \%$. Measures with variance greater than $10 \%$ were not used in the final mean calculation.

HGS was measured using a hydraulic dynamometer (SH 5001, SAEHAN Corp, Korea) which has two parallel handles which are adjustable according to the dimensions of the hands. This equipment measures the force produced by a maximum isometric contraction, which is recorded in kilograms-force (kgf) or pounds. The maximal HGS of the dominant limb was evaluated in the orthostatic posture, with the elbow flexed at $90^{\circ}$ and the other joints (shoulder and wrist) in neutral positions. Three measurements were performed and their mean was used in data analysis ${ }^{17}$.

The 5-repetition STS was used to assess lower limb muscle strength ${ }^{18}$. For the test, a standard chair (seat height $43 \mathrm{~cm}$ ) without arms and a stopwatch were used. The older adults were instructed to get up and sit five times in a chair as quickly as possible without the help of the upper limbs, which remained crossed in front of the body while performing the movement. The test began from the seated posture on the chair with the back resting on the backrest and ended when the volunteer reached this same position after getting up from the chair five times. The time taken for an attempt was used in the analyzes.

The FES-I is a self-efficacy scale that assesses the confidence of the older adults in performing daily activities without falling. Therefore, it has also been used as a measure of fear of falls ${ }^{19}$. The Brazilian version has good psychometric characteristics, with satisfactory internal consistency and reliability, with a score $\geq 23$ points associated with a history of sporadic falls ${ }^{20}$.

FRAQ assesses the perception of the older adults regarding the risk of falls ${ }^{21}$. The FRAQ score ranges from 0 to 32 points, with no cutoff established in literature. However, the higher the score, the better the older adults awareness of risk factors and fall prevention. In the FRAQ cross-cultural adaptation study for the Brazilian older adults, Lopes and Trelha ${ }^{21}$ also observed excellent reliability and internal consistency for this instrument.

Categorical variables are expressed as absolute (n) and relative (\%) frequencies, and the differences between the three groups were evaluated using Pearson's chi-square and Fisher's exact tests..

For the continuous variables, an exploratory data analysis was initially performed, including measures of normality (Shapiro-Wilk test), data distribution (Asymmetry and Kurtosis) and homogeneity of variances (Levene test). Once the normality of the data was verified, the mean, standard deviation and $95 \%$ confidence interval of the mean of all the continuous variables were calculated. For variables related to sample characterization (age, weight, height, BMI, GDS and MMSE), the differences between groups were tested using analysis of variance (ANOVA) for independent samples. For the outcome variables (TUG, HGS, STS, FES-I and FRAQ), the effects of group, time and interaction were tested by means of repeated measures covariance (ANCOVA), considering BMI as a covariate in comparisons. In case of significance, the Bonferroni and t-Student post hoc tests were used to detect inter and intragroup differences, respectively. 
The effect size of the interventions for outcome variables was also calculated using the Hedges test (g), and their values were classified as: insignificant $(<0.19)$; small (0.20-0.49); medium (0.50-0.79); large $(0.80-1.29)$ and very large (>1.30). For all statistical analyzes, the significance level was set at $\mathrm{p}<0.05$.

The present study complied with Resolution $\mathrm{n}^{\circ}$ 466/2012 of the National Health Council and was approved by the EACH|USP Ethics Committee for Research involving human beings (opinion $\mathrm{n}^{\circ}$ 1.427.294 and CAAE 51671215.6.0000.5390). After being informed about the research procedures and ethical aspects, all participants signed a Free and Informed Consent Form.

\section{RESULTS}

Of the 69 older adults included in the study, 51 completed all stages of the research. The final number of participants in the CG, PEG and MIG groups was 15, 20 and 16, respectively. The main reasons related to sample loss included not returning for reevaluation, personal problems, abandonment of activities and non-adherence to the interventions performed, i.e., a frequency below $75 \%$.

The sociodemographic data, anthropometric characteristics, history of falls, GDS and MMSE performance of the three groups are presented in Table 1. The groups did not differ for most of these variables except for weight $\left[\mathrm{F}_{(2.48)}=6.34 ; p=0.00\right]$ and BMI $\left[\mathrm{F}_{(2.48)}=6.45 ; p=0.00\right]$. The MIG group had a higher weight than the PEG $(p=0.00)$ and a higher BMI than the other groups (vs. CG, $p=0.04$ and vs. PEG, $p=0.00)$. Thus, BMI was used as a covariate in analyzes involving outcome variables. In all groups there was a higher prevalence of young older adults (mean age $67 \pm 6.2$ years), women and participants with nine or more years of study. Additionally, all groups performed well in the GDS and MMSE, with no statistical differences among them.

The comparisons between groups for the outcome variables are presented in Table 2. Regarding the physical-functional performance tests, interaction between group and time was observed. $\left[\mathrm{F}_{(2.47)}=5.02\right.$; $p=0.01]$ for TUG performance. In the intragroup analysis both groups submitted to physical training showed significant reduction in the time of this test (FEG, $p=0.02$ and MIG, $p=0.03$ ). The HGS showed the effect of time $\left[\mathrm{F}_{(1,47)}=8.80 ; p=0.00\right]$, but no statistical differences were found in the intragroup comparisons. $(p>0.05)$.

The analyzes also showed interaction between group and time $\left[\mathrm{F}_{(2.46)}=8.69 ; p=0.01\right]$ for FRAQ, with an increase in score only for the MIG group $(p=0.00)$. However, no effect was observed for STS performance and FES-I score.

Analyzes of the effect size of interventions are presented in Table 3. Both interventions had a small but significant effect on TUG time (FEG, $g=-0.25$ and MIG, $g=-0.38$ ). Although the covariance analysis did not show statistical significance for the STS test, the control situation had a significant and medium effect $(g=0.54)$ on reducing performance, while the effects of both interventions were insignificant. Multiple intervention had a large effect $(g=1.19)$ on the FRAQ score. Finally, the effects of interventions on HGS and FES-I were mostly negligible. 
Table 1. Sociodemographic characteristics, anthropometric measurements, history of falls, depressive symptoms and cognitive performance of the control (CG), physical exercise (PEG) and multiple intervention (MIG) groups. São Paulo, 2018.

\begin{tabular}{|c|c|c|c|c|}
\hline Variables & $\begin{array}{l}\text { Control Group } \\
(\mathrm{n}=15)\end{array}$ & $\begin{array}{l}\text { Physical Exercise } \\
\text { Group }(n=20)\end{array}$ & $\begin{array}{l}\text { Multiple Intervention } \\
\text { Group }(n=16)\end{array}$ & $p$-value \\
\hline \multicolumn{5}{|l|}{ Age (years) } \\
\hline Mean (standard deviation) & $67.1( \pm 6.28)$ & $67.3( \pm 5.56)$ & $68.1( \pm 6.82)$ & \multirow[t]{2}{*}{0.88} \\
\hline $95 \% \mathrm{CI}$ & $63.7-70.6$ & $64.7-69.9$ & $69.2-71.8$ & \\
\hline \multicolumn{5}{|l|}{ Sex, n $(\%)$} \\
\hline Female & $10(66.7)$ & $17(85.0)$ & $12(75.0)$ & \multirow[t]{2}{*}{0.44} \\
\hline Male & $5(33.3)$ & $3(15.0)$ & $4(25.0)$ & \\
\hline \multicolumn{5}{|l|}{ Marital Status, n (\%) } \\
\hline Married / civil partnership & $6(40.0)$ & $9(45.0)$ & $7(43.8)$ & \multirow{4}{*}{0.41} \\
\hline Single & $6(40.0)$ & $3(15.0)$ & $2(12.5)$ & \\
\hline Widowed & $2(13.3)$ & $5(25.0)$ & $6(37.5)$ & \\
\hline Divorced / Separated & $1(6.7)$ & $3(15.0)$ & $1(6.2)$ & \\
\hline \multicolumn{5}{|l|}{ Schooling (years), n (\%) } \\
\hline $1-4$ & $2(13.3)$ & $3(15.0)$ & $2(12.5)$ & \multirow{3}{*}{0.90} \\
\hline $5-8$ & $3(20.0)$ & $5(25.0)$ & $2(12.5)$ & \\
\hline$\geq 9$ & $10(67.0)$ & $12(60.0)$ & $12(75.0)$ & \\
\hline \multicolumn{5}{|l|}{ Height (m) } \\
\hline Mean (standard deviation) & $1.61( \pm 0.95)$ & $1.58( \pm 0.49)$ & $1.61( \pm 0.86)$ & \multirow[t]{2}{*}{0.42} \\
\hline $95 \% \mathrm{CI}$ & $1.56-1.67$ & $1.56-1.61$ & $1.56-1.66$ & \\
\hline \multicolumn{5}{|l|}{ Weight $(\mathrm{kg})$} \\
\hline Mean (standard deviation) & $71.0( \pm 10.4)$ & $67.0( \pm 8.69)^{*}$ & $80.0( \pm 13.6)$ & \multirow[t]{2}{*}{0.00} \\
\hline $95 \% \mathrm{CI}$ & $65.1-76.7$ & $62.7-70.8$ & $72.4-86.9$ & \\
\hline \multicolumn{5}{|l|}{ BMI (kg / m2) } \\
\hline Mean (standard deviation) & $27.4( \pm 3.80)^{*}$ & $26.4( \pm 2.57)^{*}$ & $30.6( \pm 4.31)$ & \multirow[t]{2}{*}{0.00} \\
\hline $95 \% \mathrm{CI}$ & $24.3-29.6$ & $25.2-27.6$ & $28.3-32.9$ & \\
\hline \multicolumn{5}{|l|}{ Fall history, n ( $\%)$} \\
\hline Yes & $4(26.7)$ & $2(10.0)$ & $5(31.6)$ & \multirow[t]{2}{*}{0.26} \\
\hline No & $11(73.3)$ & $18(90.0)$ & $11(78.4)$ & \\
\hline \multicolumn{5}{|l|}{ GDS (score) } \\
\hline Mean (standard deviation) & $1.93( \pm 1.59)$ & $1.80( \pm 2.28)$ & $2.06( \pm 1.98)$ & \multirow[t]{2}{*}{0.98} \\
\hline $95 \%$ CI & $1.01-2.85$ & $0.73-2.87$ & $2.06-3.12$ & \\
\hline \multicolumn{5}{|l|}{ MMSE (score) } \\
\hline Mean (standard deviation) & $26.4( \pm 2.56)$ & $25.6( \pm 2.32)$ & $26.6( \pm 2.13)$ & \multirow[t]{2}{*}{0.35} \\
\hline $95 \% \mathrm{CI}$ & $24.9-27.9$ & $25.6-27.7$ & $25.4-27.7$ & \\
\hline
\end{tabular}

$\mathrm{CI}=$ confidence interval; $\mathrm{BMI}=$ body mass index; GDS = geriatric depression scale; MMSE = Mini Mental State Examination; ${ }^{*}<<0.05 v s$. MIG. 
Table 2. Comparison between control (CG), exercise (FEG), and multiple intervention (MIG) groups for mobility, handgrip strength, lower limb strength, self-efficacy, and perception of risk factors for falls. São Paulo, 2018.

\begin{tabular}{|c|c|c|c|c|c|c|c|c|c|}
\hline \multirow[t]{2}{*}{ Variables } & \multicolumn{2}{|c|}{ Control Group $(n=15)$} & \multicolumn{2}{|c|}{$\begin{array}{l}\text { Physical Exercise Group } \\
(\mathrm{n}=20)\end{array}$} & \multicolumn{2}{|c|}{$\begin{array}{l}\text { Multiple Intervention } \\
\text { Group }(n=16)\end{array}$} & \multicolumn{3}{|c|}{$p$-value } \\
\hline & Pre & Post & Pre & Post & Pre & Post & G & $\mathrm{T}$ & GxT \\
\hline \multicolumn{10}{|l|}{ TUG (sec) } \\
\hline Mean (sd) & $7.57( \pm 0.82)$ & $7.77( \pm 1.06)$ & $7.64( \pm 1.42)$ & $7.26( \pm 1.02)^{*}$ & $7.72( \pm 1.07)$ & $7.32( \pm 0.90)^{*}$ & 0.82 & 0.10 & 0.01 \\
\hline CI 95\% & $7.12-8.03$ & $7.18-8.36$ & $6.98-8.31$ & $6.79-7.75$ & $7.15-8.29$ & $6.84-7.80$ & & & \\
\hline \multicolumn{10}{|l|}{ HGS (kgf) } \\
\hline Mean (sd) & $27.1( \pm 8.04)$ & $26.7( \pm 9.67)$ & $23.3( \pm 3.84)$ & $24.9( \pm 3.97)$ & $24.7( \pm 7.74)$ & $25.5( \pm 6.99)$ & 0.48 & 0.00 & 0.13 \\
\hline CI 95\% & $22.7-31.6$ & $21.3-32.1$ & $21.6-25.2$ & $23.1-26.8$ & $20.6-28.9$ & $21.8-29.3$ & & & \\
\hline \multicolumn{10}{|l|}{ STS (sec) } \\
\hline Mean (sd) & $11.5( \pm 2.17)$ & $12.6(1.43)$ & $12.7( \pm 4.04)$ & $12.5(2.18)$ & $12.7( \pm 2.72)$ & $12.6(1.92)$ & 0.68 & 0.29 & 0.19 \\
\hline CI 95\% & $10.3-12.7$ & $11.8-13.4$ & $10.8-14.6$ & $11.5-13.5$ & $11.3-14.2$ & $11.6-13.6$ & & & \\
\hline \multicolumn{10}{|c|}{ FES-I (score) } \\
\hline Mean (sd) & $22.6( \pm 3.76)$ & $24.5(6.19)$ & $24.2( \pm 6.22)$ & $25.4(7.53)$ & $24.7( \pm 6.23)$ & $24.6(6.51)$ & 0.65 & 0.32 & 0.51 \\
\hline CI 95\% & $20.4-24.7$ & $20.9-28.1$ & $21.3-27.1$ & 21.9-28.9 & $21.4-28.1$ & $21.1-28.1$ & & & \\
\hline \multicolumn{10}{|c|}{ FRAQ (score) } \\
\hline Mean (sd) & $22.7( \pm 2.87)$ & $24.3( \pm 2.76)$ & $23.1( \pm 3.79)$ & $23.2( \pm 3.27)$ & $20.4( \pm 4.33)$ & $25.8( \pm 4.49)^{*}$ & 0.95 & 0.44 & 0.01 \\
\hline CI 95\% & $21.1-24.4$ & $22.7-25.9$ & $21.3-24.9$ & $21.7-24.8$ & $18.1-22.7$ & $23.4-28.2$ & & & \\
\hline
\end{tabular}

$\mathrm{G}=$ group effect; $\mathrm{T}=$ effect of time; $\mathrm{GxT}=$ interaction between group and time effects; sd = standard deviation; $\mathrm{CI}=$ confidence interval; $\mathrm{TUG}$ = Timed Up and Go; HS = hand grip strength; CS = chair stand; FES-I = Falls Efficacy Scale International; FRAQ = Falls Risk Awareness Questionnaire; ${ }^{*} p<0.05$ vs. basal situation within the same group.

Table 3. Analysis of the effect size of the interventions (pre vs. post) for the control (CG), exercise (PEG) and multiple intervention (MIG) groups for mobility, handgrip strength, lower limb strength, self-efficacy, and perception about risk factors for falls. São Paulo, 2018.

\begin{tabular}{|c|c|c|c|c|c|c|c|c|}
\hline \multirow{2}{*}{ Variables } & \multirow[t]{2}{*}{ Hedges $g$} & \multicolumn{2}{|c|}{ Confidence $95 \%$} & \multirow[t]{2}{*}{ Student's t } & \multirow[t]{2}{*}{ df } & \multirow[t]{2}{*}{$p$-value } & \multirow[t]{2}{*}{ CLES } & \multirow[t]{2}{*}{ ES Classification } \\
\hline & & CI Lower & CI Upper & & & & & \\
\hline \multicolumn{9}{|l|}{ TUG (sec) } \\
\hline CG & 0.19 & -0.14 & 0.54 & 1.27 & 14 & 0.22 & 55.6 & Insignificant \\
\hline PEG & -0.25 & -0.70 & -0.06 & -2.46 & 19 & 0.02 & 57.0 & Small \\
\hline MIG & -0.38 & -0.76 & -0.04 & -2.38 & 15 & 0.03 & 60.7 & Small \\
\hline \multicolumn{9}{|c|}{ HGS (kgf) } \\
\hline CG & -0.11 & -0.87 & 0.07 & -1.81 & 14 & 0.09 & 53.1 & Insignificant \\
\hline PEG & 0.19 & -2.38 & 5.58 & 0.84 & 19 & 0.41 & 55.4 & Insignificant \\
\hline MIG & 0.10 & -1.00 & 2.60 & 0.36 & 15 & 0.36 & 52.8 & Insignificant \\
\hline \multicolumn{9}{|l|}{ STS (sec) } \\
\hline CG & 0.54 & 0.20 & 2.00 & 2.61 & 14 & 0.02 & 64.9 & Medium \\
\hline PEG & -0.04 & -1.37 & 0.97 & -0.36 & 19 & 0.73 & 51.2 & Insignificant \\
\hline MIG & -0.04 & -1.01 & 0.81 & -0.23 & 15 & 0.82 & 51.2 & Insignificant \\
\hline
\end{tabular}


Continuation of Table 3

\begin{tabular}{|c|c|c|c|c|c|c|c|c|}
\hline \multirow{2}{*}{ Variables } & \multirow[t]{2}{*}{ Hedges $g$} & \multicolumn{2}{|c|}{ Confidence $95 \%$} & \multirow[t]{2}{*}{ Student's t } & \multirow[t]{2}{*}{ df } & \multirow[t]{2}{*}{$p$-value } & \multirow[t]{2}{*}{ CLES } & \multirow[t]{2}{*}{ ES Classification } \\
\hline & & CI Lower & CI Upper & & & & & \\
\hline \multicolumn{9}{|c|}{ FES-I (score) } \\
\hline CG & 0.33 & -0.68 & 4.48 & 1.58 & 14 & 0.14 & 59.3 & Small \\
\hline PEG & 0.17 & -2.56 & 4.96 & 0.67 & 19 & 0.51 & 54.8 & Insignificant \\
\hline MIG & -0.02 & -2.55 & 2.35 & -0.09 & 15 & 0.93 & 53.8 & Insignificant \\
\hline \multicolumn{9}{|c|}{ FRAQ (score) } \\
\hline CG & 0.55 & -0.84 & 4.04 & 1.41 & 14 & 0.18 & 65.2 & Medium \\
\hline PEG & 0.03 & -1.07 & 1.27 & 0.18 & 19 & 0.86 & 50.9 & Insignificant \\
\hline MIG & 1.19 & 3.27 & 7.53 & 5.41 & 15 & 0.00 & 80.2 & Large \\
\hline
\end{tabular}

$\mathrm{CI}=$ confidence interval; $\mathrm{df}=$ degree of freedom; $\mathrm{CLES}=$ common language effect size; $\mathrm{TUG}=$ Timed $\mathrm{Up}$ and $\mathrm{G} 0$; $\mathrm{HS}=$ handgrip strength; $\mathrm{CS}=$ chair stand test; FES-I= Falls Efficacy Scale International; FRAQ= Falls Risk. Awareness Questionnaire.

\section{DISCUSSION}

Scientific evidence suggests that multimodal physical training has several health benefits for the older adults ${ }^{22}$, and is also the most recommended in the prevention of falls as an single intervention ${ }^{4}$. Although the effects of this type of training are well established, its magnitude varies widely between studies. According to the systematic review by Bouaziz et al. ${ }^{22}$, the gains from multimodal training in muscle strength and mobility ranged from 1.4 to $95 \%$ and 5.3 to $88.9 \%$, respectively. These differences may be related to several factors, including the characteristics of the subjects involved, the tests used and the physical training program itself.

In the present study an improvement of approximately $5 \%$ in mobility was observed in both groups undergoing physical training which, although small, was statistically significant. On the other hand, no changes were observed in performance in muscle strength tests after the follow-up period. It is important to remember that the older adults included in this study came from an U3A and, therefore, have some particular characteristics compared to older adults recruited from other types of services and locations. A previous study within the same U3A showed that its participants are relatively young (mean age $67 \pm 6.2$ years), have a high level of education (eight years or more of study) and are physically active $e^{23}$, corroborating the characteristics of the older adults in this research. Additionally, older adults participants of U3As tend to present good physical and functional performance ${ }^{10,23}$ and therefore, a low risk of falls.

Due to the predictive power for negative outcomes, physical-functional tests have been widely investigated in literature. Regarding the risk of future falls, a meta-analysis study suggested cutoff values for different tests $/ \mathrm{scales}^{24}$, with older adults with TUG and STS times longer than 11 and 12 seconds, respectively, considered at risk. In the same study, FES-I scores greater than 24 were also indicative of a risk of future falls. For Brazilian communitydwelling older adults, Alexandre et al. ${ }^{16}$ showed that TUG values greater than 12.47 seconds are predictive of falls, with a sensitivity of $73.7 \%$ and a specificity of $65.8 \%$. In the present study, all the groups had lower TUG values than those suggested for the risk of falls, regardless of whether international ${ }^{24}$ or Brazilian ${ }^{16}$ reference values are applied. If compared with the study by Lusardi et al. ${ }^{24}$, STS and FES-I values were borderline for risk of falls in all groups and therefore should not be disregarded.

The importance of maintaining good physical performance in old age has been demonstrated by several studies ${ }^{25-28}$. Den Ouden et al. ${ }^{25}$, in a 10-year prospective study, observed that muscle strength, associated with number of chronic diseases, age, sex (female) and socioeconomic status, was a determining factor for dependence on daily living activities in community-dwelling older adults. Similarly, Dodds et $a .^{26}$ evaluated the influence of different factors in determining future disability in middle-aged 
individuals. The variables that best fit the model were sex (female), the presence of knee osteoarthritis, use of two or more medications, being a smoker, having a high BMI and poor performance in the HGS, STS and balance tests.

The present study also revealed that early physical-functional performance tests, i.e., in middle age, increased the discriminative power of at-risk subjects in 16 years of follow-up ${ }^{16}$. These findings underscore the importance of encouraging older people to participate in multimodal physical training programs, regardless of their physical condition. Above average physical performance can act as a "physical reserve," allowing older people to respond adequately to stressful events"

The benefits of physical training alone in psychological variables, such as fear of falls, for example, are controversial. According to Whipple et al. $^{28}$, multiple interventions are most effective in reducing the fear of falls, especially when involving multimodal physical training and cognitive behavioral intervention. Freiberger et al..$^{29}$ conducted a randomized clinical trial to evaluate the effect of three different interventions on older adults with a history of falls and fear of falls. All interventions consisted of exercises of muscular resistance and balance, and were differentiated with a third component: a) strength and balance: intensity of the exercises progressively increased, b) fitness: addition of aerobic endurance and c) multiple intervention: addition of education on risk factors for falls and cognitive behavioral training. The authors observed improved lower limb mobility and strength (except for the multiple intervention group) after six months of follow-up. However, no change was observed for measures related to knowledge of risk factors and fear of falls. In contrast, Siegrist et al. ${ }^{30}$ found positive effects on balance and fear of falling in older adults in primary care after 16 weeks of multiple intervention (physical exercise combined with educational activities). As in the study by Freiberger et al. ${ }^{29}$, there was no improvement in fear of falls (FES-I) in the present work. On the other hand, the multiple intervention group showed greater knowledge about the risk factors of falls than the other groups after the intervention, contrary to the results of these authors ${ }^{29}$.
As well as other risk factors for falls recognized in literature, Moreira et al..$^{31}$ showed that poor knowledge about risk factors (assessed by the FRAQ) was also associated with the history of falls in communitydwelling older adults. The authors observed that physical-functional performance and perception of risk factors were different between older adults with and without history of falls, regardless of age. Older adults with greater knowledge about risk factors and no history of falls also had better physical-functional and cognitive performance. According to Chehuen Neto et al..$^{32}$, the older adults generally has little knowledge about falls, and does not recognize themselves as a vulnerable group. In this study, an inverse association was also observed between the perception of risk and the presence of household risk factors, suggesting lower exposure to risk of older adults with greater knowledge about falls.

In a systematic review, Campbell and Robertson ${ }^{33}$ observed that for older adults in the community, isolated interventions are as effective as combined interventions. Factors contributing to the lack of additional effects of combined interventions include the poor adherence of the older adults, difficulties in implementing programs for the general population, and lack of recognition of the importance of programs for the older adults. According to Hill et al..$^{34}$, the older adults do not believe that such an approach can reduce falls and therefore strategies aimed at raising awareness that falls can be preventable rather than unpredictable, thus contradicting the idea that nothing can be done in terms of falls, may be a facilitating measure for adherence to prevention programs and, consequently, for the prevention of future events. In this sense, Schepens et al. ${ }^{9}$ found positive effects of educational methods for the prevention of falls, and improved knowledge of the older adults regarding risk and preventive behavior. The present study partly agrees with these authors ${ }^{9}$, since the multiple intervention group, which participated in the educational activities, had increased knowledge about risk factors. However, the impact of these activities on preventive behavior has not been evaluated.

Finally, the present study has some limitations that should be pointed out. Initially, the study was designed as a randomized clinical trial, also 
involving a group submitted only to an educational intervention. However, the older adults allocated in this group had low adherence to the proposed activities, making it clear to the researchers that the greatest interest of the older adults was to participate in physical training groups.

Another important limitation concerns the study sample. Older adults participants in U3As are very active and, therefore, perform well in physical and functional tests. In combination with poorly sensitive testing for younger, highly-performing older adults, the results of the interventions may have been underestimated. According to Bergquist et al. ${ }^{35}$, most physical-functional tests have a ceiling effect on younger, more active older people, making early identification of declines difficult. The Community Balance and Mobility scale seems to be a more sensitive option for this group, although it has not yet been validated for the Brazilian population. Finally, due to the involvement in different activities within the U3A, the influence of other activities on the variables studied can also not be excluded, thus characterizing a significant study bias.

\section{CONCLUSION}

The multiple intervention, involving physical exercises and educational activities, brought additional benefits to the older adults participants of the Open University for the Third Age studied. In addition to improved balance, older adults in the multiple intervention group showed greater knowledge about risk factors for falls after the followup period, which may be helpful in implementing strategies for reducing risk behaviors in daily life.

Edited by: Ana Carolina Lima Cavaletti

\section{REFERENCES}

1. Ambrose AF, Paul G, Hausdorff JM. Risk factors for falls among older adults: a review of the literature. Maturitas. 2013;75(1):51-61.

2. Florence CS, Bergen G, Atherly A, Burns E, Stevens J, Drake C. Medical costs of fatal and nonfatal falls in older adults. J Am Geriatr Soc. 2018;66(4):693-8.

3. Pimentel WRT, Pagotto V, Stopa SR, Hoffmann MCCL, Andrade FB, Souza Junior PRB, et al. Falls among Brazilian older adults living in urban areas: ELSI-Brazil. Rev Saúde Pública. 2018;52(Suppl 2):1-9.

4. Gillespie LD, Robertson MC, Gillespie WJ, Sherrington C, Gates S, Clemson LM, et al. Interventions for preventing falls in older people living in the community. Cochrane Database Syst Rev. 2012;(9):1-4.

5. Sherrington C, Michaleff ZA, Fairhall N, Paul SS, Tiedemann A, Whitney J, et al. Exercise to prevent falls in older adults: an updated systematic review and meta-analysis. Br J Sports Med. 2017;51(24):1750-8.

6. Cheng P, Tan L, Ning P, Li L, Gao Y, Wu Y, et al. Comparative effectiveness of published interventions for elderly fall prevention: a systematic review and network meta-analysis. Int J Environ Res Public Health. 2018;15(3):498-14.

7. Guirguis-Blake JM, Michael YL, Perdue LA, Coppola EL, Beil TL. Interventions to prevent falls in older adults. JAMA. 2018;319(16):1705-12.
8. Goodwin VA, Abbott RA, Whear R, Bethel A, Ukoumunne OC, Thompson Coon J, et al. Multiple component interventions for preventing falls and fallrelated injuries among older people: systematic review and meta-analysis. BMC Geriatr. 2014;14(1):1-8.

9. Schepens SL, Panzer V, Goldberg A. Randomized controlled trial comparing tailoring methods of multimedia-based fall prevention education for community-dwelling older adults. Am J Occup Ther. 2011;65(6):702-9.

10. Vieira ND, Testa D, Ruas PC, Salvini TF, Catai AM, Melo RC. The effects of 12 weeks Pilates-inspired exercise training on functional performance in older women: a randomized clinical trial. J Bodywork Mov Ther. 2017;21(2):251-8.

11. Almeida OP, Almeida SA. Confiabilidade da versão brasileira da Escala de Depressão em Geriatria (GDS) versão reduzida. Arq Neuropsiquiatr. 1999;57(2B):421-6.

12. Paradela E, Lourenço RA, Veras RP. Validação da escala de depressão geriátrica em um ambulatório geral. Rev Saúde Pública. 2005;39(6):918-23.

13. Brucki SMD, Nitrini R, Caramelli P, Bertolucci PHF, Okamoto IH. Suggestions for utilization of the mini-mental state examination in Brazil. Arq NeuroPsiquiatr. 2003;61(3B):777-81. 
14. de Melo DM, Barbosa AJG. O uso do Mini-Exame do Estado Mental em pesquisas com idosos no Brasil: uma revisão sistemática. Ciênc Saúde Colet. 2015;20(12):3865-76.

15. Northgraves MJ, Hayes SC, Marshall P, Madden LA, Vince RV. The test-retest reliability of four functional mobility tests in apparently healthy adults. Isokinet Exerc Sci. 2016;24:171-9.

16. Alexandre TDS, Corona LP, Nunes DP, Santos JLF, Duarte YAO, Lebrão ML. Gender differences in incidence and determinants of disability in activities of daily living among elderly individuals: SABE study. Arch Gerontol Geriatr. 2012;55(2):431-7.

17. Boadella JM, Kuijer PP, Sluiter JK, Frings-Dresen MH. Effect of self-selected handgrip position on maximal handgrip strength. Arch Phys Med Rehabil. 2005;86(2):328-31.

18. Mehmet H, Yang AWH, Robinson SR. What is the optimal chair stand test protocol for older adults?: a systematic review. Disabil Rehabil [Internet]. 2019 [acesso em 10 jul. 2019]. [Epub ahead of print]. Disponível em: https://www.tandfonline.com/doi/ full/10.1 n080/09638288.2019.1575922

19. Marques-Vieira CMA, Sousa LMM, Severino S, Sousa L, Caldeira S. Cross-cultural validation of the falls efficacy scale international in elderly: Systematic literature review. J Clin Geront Geriatr. 2016;7(3):72-6.

20. Camargos FFO, Dias RC, Dias JMD, Freire MTF. Cross-cultural adaptation and evaluation of the psychometric properties of the Falls Efficacy Scale - International Among Elderly Brazilians (FES-IBRAZIL). Rev Bras Fisioter. 2010;14(3):237-43.

21. Lopes AR, Trelha CS. Translation, cultural adaptation and evaluation of the psychometric properties of the Falls Risk Awareness Questionnaire (FRAQ): FRAQBrazil. Rev Bras Fisioter. 2013;17(6):593-605.

22. Bouaziz W, Lang PO, Schmitt E, Kaltenbach G, Geny B, Vogel T. Health benefits of multicomponent training programmes in seniors: a systematic review. Int J Clin Practice. 2016;70(7):520-36.

23. Melo RC, Santos CCN, Yassuda MS, Domingues MARC, Lopes A, Meire C. Desempenho físicofuncional e nível de atividade física de participantes da Universidade Aberta à Terceira Idade da Escola de Artes Ciências e Humanidades da Universidade de São Paulo (UnATI EACH-USP). Rev Kairós. 2012;15(7):129-54.

24. Lusardi MM, Fritz S, Middleton A, Allison L, Wingood M, Phillips E, et al. Determining risk of falls in community dwelling older adults. J Geriatr Phys Ther. 2017;40(1):1-36.
25. den Ouden MEM, Schuurmans MJ, Mueller-Schotte $\mathrm{S}$, van der Schouw YT. Identification of high-risk individuals for the development of disability in activities of daily living. A ten-year follow-up study. Exp Gerontol. 2013;48(4):437-43.

26. Dodds RM, Kuh D, Sayer AA, Cooper R. Can measures of physical performance in mid-life improve the clinical prediction of disability in early old age? Findings from a British birth cohort study. Exp Gerontol. 2018;110:118-24.

27. Blain H, Carriere I, Sourial N, Berard C, Favier F, Colvez A, et al. Balance and walking speed predict subsequent 8-year mortality independently of current and intermediate events in well-functioning women aged 75 years and older. J Nutr Health Aging. 2010;14(7):595-600.

28. Whipple MO, Hamel AV, Talley KMC. Fear of falling among community-dwelling older adults: a scoping review to identify effective evidence-based interventions. Geriatr Nurs. 2018;39(2):170-7.

29. 29. Freiberger E, Häberle L, Spirduso WW, Zijlstra GAR. Long-term effects of three multicomponent exercise interventions on physical performance and fall-related psychological outcomes in communitydwelling older adults: a randomized controlled trial. J Am Ger Soc. 2012;60(3):437-46.

30. Siegrist M, Freiberger E, Geilhof B, Salb J, Hentschke $\mathrm{C}$, Landendoerfer $\mathrm{P}$, et al. Fall prevention in a primary care setting. Dtsch Arztebl Int. 2016;113(21):365-72.

31. Moreira NB, Rodacki ALF, Pereira G, Bento PCB. Does functional capacity, fall risk awareness and physical activity level predict falls in older adults in different age groups? Arch Gerontol Geriatr. 2018;77:57-63.

32. Chehuen Neto JA, Braga NAC, Brum IV, Gomes GF, Tavares PL, Silva RTC, et al. Percepção sobre queda e exposição de idosos a fatores de risco domiciliares. Ciênc Saúde Colet. 2018;23(4):1097-104.

33. Campbell AJ, Robertson MC. Rethinking individual and community fall prevention strategies: a metaregression comparing single and multifactorial interventions. Age Ageing. 2007;36(6):656-62.

34. Hill KD, Day L, Haines TP. What factors influence community-dwelling older people's intent to undertake multifactorial fall prevention programs? Clin Interv Aging. 2014;9:2045-53.

35. Bergquist R, Weber M, Schwenk M, Ulseth S, Helbostad JL, Vereijken B, et al. Performance-based clinical tests of balance and muscle strength used in young seniors: a systematic literature review. BMC Geriatr [Intermet]. 2019. [acesso em 10 jul. 2019];19(1):1-14. Disponível em: https://bmcgeriatr. biomedcentral.com/articles/10.1186/s12877-018-1011-0 